\title{
Analysis of Nanoemulsion Coatings
}

\section{Chun-Jen $\mathbf{W u}^{1}$ and Agnes Ostafin ${ }^{2 *}$}

${ }^{1}$ Department of Materials Science, Nanolnstitute, University of Utah, Salt Lake City UT 84112, USA

${ }^{2}$ Department of Chemical Engineering, Department of Bioengineering, Nanolnstitute, University of Utah, Salt Lake City UT 84112, USA

\begin{abstract}
This work described a method to understand the dynamics of nanoemulsion coatings. Using a combination of zeta potential measurements, absorption spectroscopy, and fluorescence quenching it is possible to understand how the conformation of molecules at the nanoemulsion surface change as a function of loading. Conformational changes as a function of $\mathrm{pH}$, temperature and other external inputs are known, but those as a function of coating composition density have not been studied in these systems. The main result is that more coating molecules do not necessarily generate a less permeable coating. The concentration-dependent conformational changes of individual coating molecules has to be taken into consideration, because in some cases it can create unexpectedly highly porous coatings.
\end{abstract}

Keywords: Nanoemulsion; Nanocoatings

\section{Introduction}

Stability and performance control of nanoemulsions and liposomes is enhanced when they are stabilized by surfactants and coated with other molecules. Coatings can be selected from a wide range of materials, most often proteins or polymers, to provide various functions such as chemical responsiveness [1-4], diffusional gating [5-7], or target recognition function [8-11]. However, this process is largely hit or miss, since there is no guarantee that these molecules retain all of their physico-chemical characteristics once tethered to the particle. Also, due to concentration, lipid, and surface charge dependent denaturation [12-15] unanticipated activation of the immune system or other undesired interaction with blood and tissues [16-20] may occur.

It would be helpful to be able to know at a molecular level what happens to coating molecules once they are attached onto the nanoemulsions or liposomes. This information could be used to explain unanticipated behaviors as well as guide mitigating approaches. Computational approaches are too unwieldy as these are complex systems with independent phase behavior at more than one length scale. Experimentally, there are few tools that can provide clean data for interpretation that do not disrupt these metastable systems. Electromagnetic radiation scattering by the relatively large nanoemulsion particles complicates spectroscopy [21-23], Fourier Transform infrared spectroscopy [24-27] and neutron scattering [28-30].

Dynamic light scattering coupled with zeta potential measurements provide information about the impact of molecular level changes at the particle surface on diffusivity as well as overall phase behavior of the suspension. In this work, we evaluated whether this method has potential to help better understand the conformational status of molecules used as a coating. For this purpose, two model compounds, chitosan and polylysine, were utilized. The chitosan is a large molecular weight polysaccharide while polylysine is a small molecular weight polypeptide. These molecules behave differently in terms of phase behavior as a function of temperature, concentration, and $\mathrm{pH}$ and can provide insight about the range of effects that might be expected.

\section{Experimental Methods}

\section{Materials}

L-a-phosphatidylcholine (lecithin) from soybean and 1,2-dioleoyl$s n$-glycero-3-phosphate (sodium salt) (DOPA) were purchased from Avanti ${ }^{\circledR}$. Perfluorocarbon 1-bromoperfluorooctane (PFOB) was purchased from Oakwood Products, Inc. Sodium phosphate monobasic $\left(\mathrm{NaH}_{2} \mathrm{PO}_{4} \cdot \mathrm{H}_{2} \mathrm{O}\right)$ was acquired from Fisher Scientific. Sodium phosphate dibasic, heptahydrate $\left(\mathrm{Na}_{2} \mathrm{HPO}_{4} .7 \mathrm{H}_{2} \mathrm{O}\right)$, acetic acid, $\mathrm{HCl}$ and $\mathrm{NaOH}$ were acquired from Mallinckrodt. Chitosan (CS) $\left(\mathrm{M}_{\mathrm{r}} \sim 150,000 \mathrm{~g} / \mathrm{mol}\right)$ was acquired from Fluka. Poly-L-lysine (PLL) with average molecular weight from $500 \mathrm{~g} / \mathrm{mol}$ to $2000 \mathrm{~g} / \mathrm{mol}$, MES buffer, Trypan Blue assay, Cibacron Brilliant Red 3B-A assay, glycine were acquired from Sigma-Aldrich ${ }^{\circledR}$. Ethanol was obtained from PharmcoAAPER. Deionized water 18.20 M $\Omega$-cm was acquired from a Barnstead MicroPure ST (Thermo Scientific).

\section{Emulsion preparation}

Lecithin, DOPA, or Lecithin/DOPA mixture was weighed according to Table 1 and added to phosphate buffer (prepared by mixing $2 \mathrm{ml}$ of $0.2 \mathrm{M} \mathrm{NaH}_{2} \mathrm{PO}_{4} \cdot \mathrm{H}_{2} \mathrm{O}$ and $298.68 \mathrm{ml}$ of $0.2 \mathrm{M} \mathrm{Na}_{2} \mathrm{HPO}_{4} \cdot 7 \mathrm{H}_{2} \mathrm{O}$ with $859.25 \mathrm{ml}$ E-pure water, $18 \mathrm{M} \Omega \mathrm{cm}$ resistivity) in a clean, $20 \mathrm{ml}$, glass vial. To the mixture was added a designated amount of PFOB (shown in Table 1) and the resulting coarse emulsion magnetically stirred at $1200 \mathrm{rpm}$ for $30 \mathrm{~min}$ to disperse. The emulsions were extruded five times at room temperature through a $200 \mathrm{~nm}$ pore size polycarbonate membrane (Nucleopore Track-Etch membrane, Whatman) using 10 ml Thermobarrel LIPEX extruder (Northern Lipids, Burnaby, BC,

\begin{tabular}{|l|l|l|l|l|l|l|l|}
\hline Component (amount) & $\mathbf{1}$ & $\mathbf{2}$ & $\mathbf{3}^{\star}$ & $\mathbf{4}$ & $\mathbf{5}$ & $\mathbf{6}$ & $\mathbf{7}$ \\
\hline Lecithin (mmoles) & 2.33 & 2.31 & 2.28 & 2.23 & 2.21 & 2.16 & 0 \\
\hline DOPA (mmoles) & 0.00 & 0.02 & 0.05 & 0.10 & 0.12 & 0.17 & 2.33 \\
\hline PFOB (ml) & 1.60 & 1.60 & 1.60 & 1.60 & 1.60 & 1.60 & 1.60 \\
\hline Phosphate Buffer (ml) & 4.24 & 4.24 & 4.24 & 4.24 & 4.24 & 4.24 & 4.24 \\
\hline $\begin{array}{l}\text { DOPA (mol\%) total } \\
\text { surfactants }\end{array}$ & 0.0 & 0.9 & 2.2 & 4.3 & 5.2 & 7.3 & 100 \\
\hline
\end{tabular}

*Sample 3 was selected for subsequent studies. Rationale is found in the text.

Table 1: Various compositions of DOPA-lecithin emulsions used in this work.

*Corresponding author: Agnes Ostafin, Department of Chemical Engineering, Department of Bioengineering, Nanolnstitute, University of Utah, Salt Lake City UT 84112, USA, Tel: 8016983955; E-mail: a.ostafin@utah.edu

Received November 22, 2016; Accepted December 07, 2016; Published December 14, 2016

Citation: Wu CJ, Ostafin A (2016) Analysis of Nanoemulsion Coatings. J Nanomed Nanotechnol 7: 410. doi: 10.4172/2157-7439.1000410

Copyright: (C) 2016 Wu CJ, et al. This is an open-access article distributed under the terms of the Creative Commons Attribution License, which permits unrestricted use, distribution, and reproduction in any medium, provided the original author and source are credited. 
Canada), using 500 psi compressed air pressure. Nanoemulsions were characterized by light scattering and zeta potential as described below.

\section{Coating}

A solution of $30 \mu \mathrm{M}$ CS was made by mixing $100 \mathrm{mg} \mathrm{CS}, 1 \mathrm{ml}$ acetic acid and $200 \mu \mathrm{l}$ ethanol with $18.8 \mathrm{ml}$ water $(\mathrm{pH} \sim 3.5)$. A solution of $4 \mathrm{mM}$ PLL solution was made by adding $100 \mathrm{mg}$ PLL into $19.9 \mathrm{ml}$ water $(\mathrm{pH} \sim 6.5)$. Sixty-seven $500 \mu \mathrm{l}$ aliquots of emulsion sample 3 (2\% DOPA/98\% Lecithin) were prepared, diluted to 2.5 times of the original volume using E-pure water and aliquoted into $20 \mathrm{ml}$ clean glass vials. Different amounts of coating material were added to each nanoemulsion aliquot to form final coating concentrations indicated below (Table 2), followed by stirring at $150 \mathrm{rpm}$ for 30 minutes at room temperature. Aliquots at each of the coating concentrations were incubated for 1 hour with stirring at 150 RPM at one of the following temperatures before measurement: $273 \mathrm{~K}, 278 \mathrm{~K}, 283,288 \mathrm{~K}, 293 \mathrm{~K}$, $298 \mathrm{~K}$ and $310 \mathrm{~K}$. Besides these samples, nanoemulsion aliquots for incubation at $298 \mathrm{~K}$ were prepared in duplicate and one set was titrated to each of the following $\mathrm{pH}: 3.51,3.57,3.62,3.71,6.95,7.02,7.08,7.12$, and 7.19 before incubation.

\section{Characterization}

pH: To measure $\mathrm{pH}$, a $\mathrm{pH}$ meter (NIST ACCMT AR60, Fisher Scientific) and micro $\mathrm{pH}$ electrode (Orion 8220BNWP, Thermo Scientific) were used. All $\mathrm{pH}$ measurements were made at room temperature.

Dynamic light scattering (DLS) and zeta potential: Size distribution and zeta potential analysis of uncoated and coated emulsions was performed using Zetasizer Nano ZEN3600 (Malvern Instruments, Malvern, Worcestershire, UK). Ten $\mu$ l samples of the prepared mixtures described in Tables 1 and 2 were diluted in disposable polystyrene cuvettes by $1 \mathrm{ml}$ E-pure water also at the indicated temperatures. The PFOB refractive index (1.3) and baseline absorbance at the instrument laser wavelength of $633 \mathrm{~nm}(0.01)$, viscosity of water $(0.8872 \mathrm{cP})$, and refractive index of water $(1.330)$ were used in the instrument program set up. For zeta potential measurements disposable polycarbonate zeta potential cells made with gold-plated beryllium copper electrodes (Malvern) were used. The cuvettes were pre-rinsed with E-pure water, and filled with $10 \mu \mathrm{l}$ of diluted sample as described above. Water dielectric constant of 80 was used in the set up.

UV-Vis: UV Mini 1240 spectrophotometer (Shimadzu Scientific Instruments Columbia, MD) was used for colorimetric determination. All the samples measured were prepared in $1.5 \mathrm{ml}$ aliquots without dilution in $1 \mathrm{~cm}$ pathlength quartz cuvette. Samples were isolated from light during preparation using aluminum foil. The wavelength range of measurement was from $400 \mathrm{~nm}$ to $700 \mathrm{~nm}$.

\begin{tabular}{|l|l|l|}
\hline c & Conc. $(\mathbf{m M})$ & Temperature $(\mathbf{K})$ \\
\hline PLL & 1.0 & \\
\hline & 0.8 & \\
\hline & 0.6 & $273,278,283,288,293$ and 298 \\
\hline & 0.4 & \\
\hline CS & 0.2 & \\
\hline & 10.4 & \\
\hline & 8.3 & $273,278,283,288,293,298$ and 310 \\
\hline & 6.3 & \\
\hline & 4.2 & \\
\hline
\end{tabular}

Table 2: Various mixing compositions for coated DOPA-lecithin emulsions.
Estimated concentration of nanoemulsion particles: MarvinSpace (ChemAxon, Hungary) was used to visualize the molecular structure of the lipids used in the nanoemulsion and estimate the head group size of DOPA $(\sim 5 \AA)$ and lecithin $(\sim 8 \AA)$. Knowing the mean emulsion particle size from DLS measurement, the mean fluid volume of PFOB per particle was calculated. Knowing the total amount of PFOB used and assuming no losses, the estimated concentration of emulsion particles used in each experimental sample was estimated to be 68.4 $\mathrm{nM}$ per each $5 \mathrm{ml}$ of sample prepared.

Colorimetric and thermodynamic determinations: Spectrophotometric determination of unattached PLL after incubation of the nanoemulsion aliquots with coating material at various concentration were performed using the Trypan Blue (TB) assay $[31,32]$. This negatively charged dye interacts with positively charged PLL to form a precipitate. After centrifugation, the loss in intensity of blue color at $578 \mathrm{~nm}$ is used as a measure of the amount of free PLL in the suspension mixture and the amount attached to the nanoparticles is calculated assuming that no dye was adhered to the walls of container used. The system was calibrated using PLL solutions prepared in 0.1 M MES with $0.15 \mathrm{M} \mathrm{NaCl}$ at $\mathrm{pH} 6.5$ that were serially diluted between $0-14.4 \mu \mathrm{M}$. The assay solution consisting of $1.1 \mathrm{mM}$ TB in $0.1 \mathrm{M}$ MES buffer was mixed with the analyte solution, placed at $37^{\circ} \mathrm{C}$ for one hour and then centrifuged at $8000 \mathrm{rpm}$ for $20 \mathrm{~min}$. The absorbance at 578 $\mathrm{nm}$ in the supernatant solution was measured by UV/Vis spectrometry and correlated to the concentration of PLL solution.

The dye Cibacron Brilliant Red 3B-A (also called as Reactive Red $4, \mathrm{CB})$ was utilized for the quantitative determination of unattached chitosan. The reaction of negatively charged dye and positively charged amine groups of chitosan causes a bathochromic (i.e. red) shift of the absorption peak to $575 \mathrm{~nm}$ from $536 \mathrm{~nm}$ [33-35]. To calibrate, an assay solution consisting of $5 \mathrm{ml}$ of $0.75 \mu \mathrm{M} \mathrm{CB}$ solution mixed with $95 \mathrm{ml}$ buffer solution $(81 \mathrm{ml}$ solution of $0.1 \mathrm{M}$ glycine and $0.1 \mathrm{M} \mathrm{NaCl}$ added into $19 \mathrm{ml} 0.1 \mathrm{M} \mathrm{HCl}$ at $\mathrm{pH}$ 3.5) was used. The concentrations of diluted CS solution from 1.7 to $2.7 \mu \mathrm{M}$ were used for standard curve, and the peak shift correlated with the CS concentration.

To perform these assays, PLL and CS coated emulsions prepared with various concentrations of PLL and CL at the indicated temperatures were centrifuged at the same temperature at $3000 \mathrm{rpm}$ for $20 \mathrm{~min}$ to pellet the denser nanoemulsion particles (density $\sim 2 \mathrm{~g} / \mathrm{ml}$ ) [36], and the supernatant collected for analysis. To accommodate the narrow analysis range of the two assays, the supernatants containing unattached PLL were diluted to 50 times by ultrapure water and those with unattached CS, 4, 3, 2 and 1 times by ultrapure water.

Using the results of obtained by the above method, the temperaturedependent equilibrium constant and associated Gibbs free energy were calculated using:

$$
\Delta \mathrm{G}=\Delta \mathrm{H}-\mathrm{T} \Delta \mathrm{S}
$$

$$
k_{b}=\frac{[\text { Bound coating material }]}{[\text { unbound coating materials }][\text { unbound emulsions }]}
$$

Where the terms in brackets are the bound and unbound concentration of PLL and CS obtained spectrophotometrically and:

$\mathrm{R}$ is the gas constant $\left(8.314 \mathrm{~kJ} / \mathrm{mol}^{-1} \mathrm{~K}^{-1}\right)$

$\mathrm{T}$ is absolute temperature)

$\mathrm{K}$ is the equilibrium constant 
The Van't Hoff equation was used to graphically obtain the enthalpy and entropy:

$$
\ln K=\frac{-\Delta H}{R T}+\frac{\Delta S}{R}
$$

Where: $\mathrm{H}$ is the enthalpy $(\mathrm{kJ} / \mathrm{mol})$

\section{$\mathrm{S}$ is the entropy $(\mathrm{J} / \mathrm{mol} / \mathrm{K})$}

Fluorescence quenching: Fluorescence quenching measurements were used to evaluate the permeability of the PLL/CS coatings. Perfluorocarbon nanoemulsions containing $4 \mathrm{mM}$ pyrene [37] were coated with PLL and CS as usual. Pyrene is poorly soluble in water [38]. The emulsion was aliquoted to five times volume and $5.0 \times 10^{-2}$, $2.0 \times 10^{-2}, 1.0 \times 10^{-2}$ and $5 \times 10^{-3} \mathrm{M}$ of KI stock solution added to form final iodide concentrations of 3.75, 7.5, 15 and $37.5 \mathrm{mM}$. Fluorescence intensity measurements at $394 \mathrm{~nm}\left(\lambda_{\mathrm{ex}}=337 \mathrm{~nm}\right)$ were taken before the addition of $\mathrm{KI}\left(\mathrm{I}_{0}\right)$ and at 30 minutes after mixing with KI. Data were plotted according to the Stern-Volmer equation as $\mathrm{I}_{0} / \mathrm{I}$ versus [Q] to determine the efficiency of fluorescent quenching [39].

$$
\mathrm{I}_{0} / \mathrm{I}=1+\mathrm{K}_{\mathrm{S}-\mathrm{V}}[\mathrm{Q}]=1+\mathrm{k}_{\mathrm{q}} \tau_{0}[\mathrm{Q}]
$$

\section{Where:}

$\mathrm{I}_{0}$ is the initial fluorescence intensity of pyrene in absence of iodide ions

I is the fluorescent intensity of pyrene quenched by a known amount of iodide

$\mathrm{K}_{\mathrm{S}-\mathrm{V}}$ is the Stern-Volmer constant $\left(\mathrm{M}^{-1}\right)$

$\mathrm{k}_{\mathrm{q}}$ is the bimolecular quenching rate constant $\left(\mathrm{M}^{-1} \mathrm{~S}^{-1}\right)$

$\tau_{0}$ is the excited state lifetime in absence of the quencher

$[\mathrm{Q}]$ is the iodide ion concentration $(\mathrm{M})$

The ratios of $\mathrm{I}_{0} / \mathrm{I}$ versus $[\mathrm{Q}]$ for PLL and CS coated emulsion were plotted and the trend lines for the data points were fitted by linear regression.

\section{Results and Discussion}

The first step involved in coating nanoemulsions is to ensure that the nanoemulsion presents a stable platform with a surface charge that is attractive to the coating material. Then the nanoemulsion particle acts as a seed surface for coating, even at coating molecule concentrations well below its precipitation point. Therefore, prior to coating, the intrinsic property of the nanoemulsions prepared with lecithin and DOPA were characterized by zeta potential and light scattering as a function of lipid composition. The intention was to identify an optimal composition for further study.

Lecithin-only stabilized nanoemulsions (LONE) had a mean zeta potential of $-30 \mathrm{mV}$ and DOPA-only stabilized nanoemulsions (DONE) nearly $-120 \mathrm{mV}$. Since zeta potential is an ensemble property, admixtures of LONE and DONE show a linearly decreasing mean zeta potential as the $\%$ of DOPA-only stabilized nanoemulsions is increased (Figure 1A solid circles) but a relatively constant mean diameter (Figure 1B solid circles). This behavior suggests that these nanoemulsions are stable and independent of one another.

The zeta potential of LONE is too small to ensure rapid seeding of the surface coating. DONE could be used but the extremely high zeta potential makes fine control over the deposition process difficult. Also, a high degree of hydrogen bonding between emulsion particles at high concentrations leads to a hard-to-handle, high viscosity suspension.
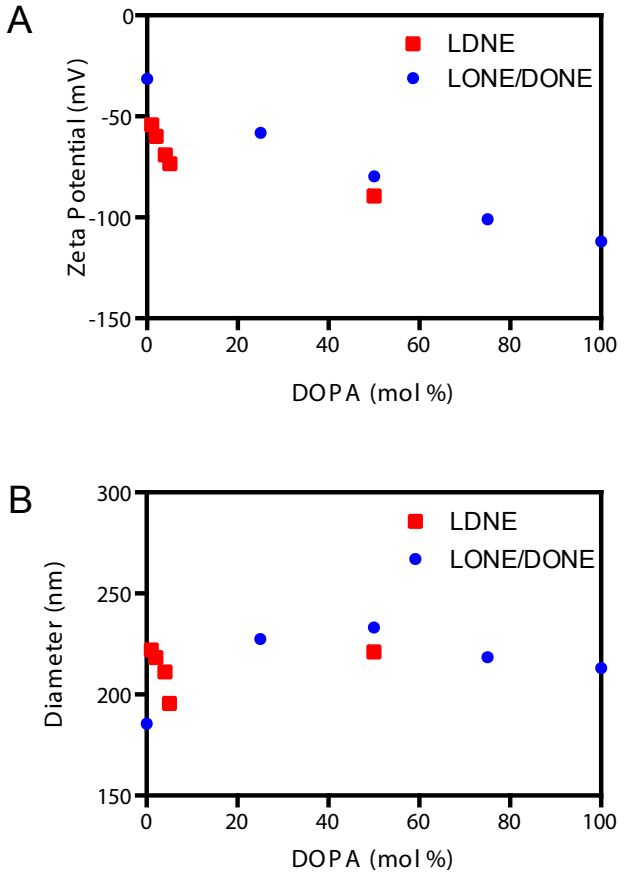

Figure 1: (A) The zeta potential of admixtures of lecithin-only stabilized nanoemulsions (LONE) and DOPA-only stabilized nanoemulsions (DONE) (blue dots) and nanoemulsions made with pre-mixed lecithin and DOPA (lecithin-DOPA stabilized nanoemulsions, LDNE) (red squares) (B) The mean diameters of the nanoemulsions. The error bar range is from $\pm 10 \mathrm{~nm}$ to \pm 25 $\mathrm{nm}$. All the measurements were done with three repeats $(n=3)$.

Instead nanoemulsions prepared with a mixture of lecithin and DOPA (LDNE) were examined. LDNE nanoemulsions exhibit a more complex zeta potential profile with increasing DOPA concentration. The measured zeta potential drops rapidly with added DOPA (Figure $1 \mathrm{~A}$ open squares), stabilizing at about $-70 \mathrm{mV}$. The DOPA and lecithin interact with each other to neutralize the positive charge of DOPA, and reduce the particle mean diameter by allowing a tighter packing of the lipid head groups (Figure 1B open squares). The plateauing of the zeta potential at around $-70 \mathrm{mV}$ suggests a limitation in the amount of DOPA that can mix with lecithin to form a single emulsion particle of given size range. Because both DOPA and lecithin have at least one unsaturated carbon bond, their hydrophobic tails are kinked and therefore would limit the extent of lipid packing possible. Any excess DOPA could form small liposomes, which would reduce the measured mean size and contribute to the overall zeta potential, but in a less dramatic way, since twice as many lipids are consumed to form a liposome of comparable size to a nanoemulsion.

Sample 3 in Table 1 (2.2 mol\% DOPA) was selected for the remainder of the study. This composition provides a reasonable zeta potential for stable seeding of the coating, a reasonable suspension viscosity despite a high particle suspension concentration, and minimized the amount of DOPA needed.

The next step in nanoemulsion coating is to establish the optimal conditions for applying the coating material. Parameters of relevance include the concentration of particles and coating material, temperature, and $\mathrm{pH}$. Keeping concentration of particles constant, we explored the effects of $\mathrm{pH}$, concentration of coating material, and temperature in order to narrow the range and focus on the conditions 
that lead to most efficient coating and to study the coating molecule's properties during and after coating.

The isoelectric point of the lipid head groups of LDNE in water was determined by measuring the zeta potential of the nanoemulsion as a function of $\mathrm{pH}$. The zeta potential switches from about $5 \mathrm{mV}$ to about $-30 \mathrm{mV}$ at $\sim \mathrm{pH} 6.9$ for the nanoemulsions for all temperatures between $273 \mathrm{~K}$ and $310 \mathrm{~K}$ (Figure 2). The mean diameter of the particles in the suspension was stable over this range except at $310 \mathrm{~K}$ where there may be a $\mathrm{pH}$ dependent re-phasing of the system (Figure 2). From these results one would expect the coating to be least stable near the isoelectic

A

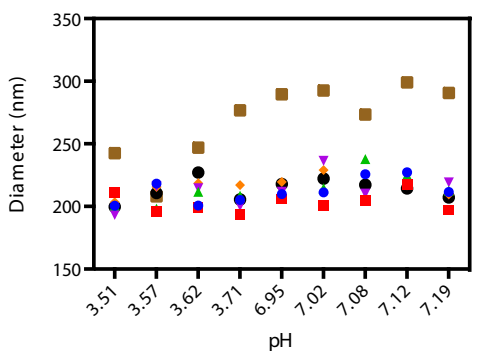

B

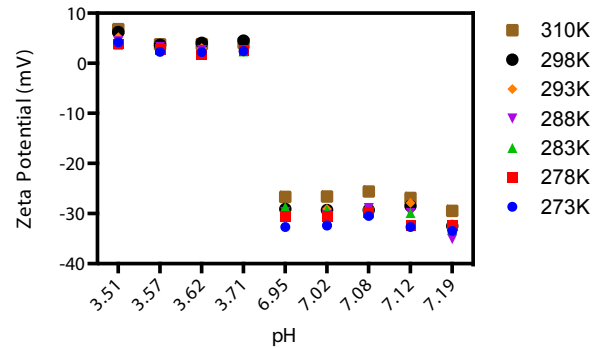

Figure 2: LDNE at various $\mathrm{pH}$ and temperature (A) size (B) zeta potential.

A

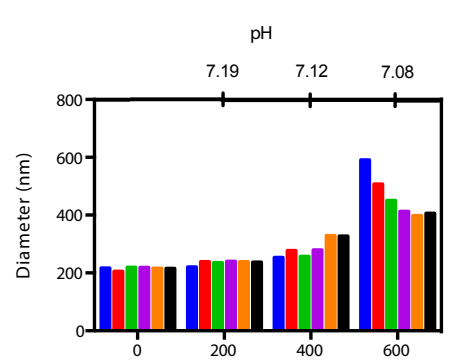

C

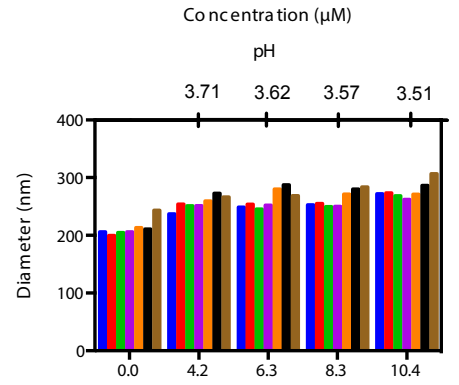

Concentration $(\mu \mathrm{M})$ point, and depending on the charge of the coating molecule either the higher or lower range of $\mathrm{pH}$ values would be preferred.

The effect of temperature and $\mathrm{pH}$ on the efficiency of coating of LDNE prepared (without further $\mathrm{pH}$ titration) with PLL and CS was examined by measuring mean diameter, zeta potential of the LDNE as a function of added coating material (Figure 3 ) and the amount of unbound coating material (Figure 4).

In general, the mean diameter of the coated LDNE increases with increasing amount of coating material added. The zeta potential becomes increasingly less negative as more PLL is added, plateauing at around $-10 \mathrm{mV}$. The same is true for CS coating, except there is a clear temperature dependence of the magnitude of this increase, with the highest temperatures (i.e. $310 \mathrm{~K}$ ) having the least change in zeta potential with CS concentration. The zeta potential difference between Figure $3 \mathrm{~B}$ and $3 \mathrm{D}$ can be interpreted due to $\mathrm{pH}$ sensitive phosphate group on the head of surfactants. The relation of $\mathrm{pH}$ and zeta potential of uncoated nanoemulsion can be viewed in Figure 2B.

Conventionally when a plateau in both particle size or zeta potential is observed, it is assumed that the particle surface is completely covered. From the data in Figure $3 \mathrm{~A}$ and $3 \mathrm{C}$ is likely that the particle size is no longer changing, and the slight trend upward may be due to changes in refractive index of the solution as the higher concentrations. To determine whether this is the case, a colorimetric method was used to determine the actual concentration of bound and unbound PLL and CS in Figures 4 and 5. For PLL the amount of bound PLL increased with increasing amount of PLL used, and decreased with increasing amount of CS (Figure 5). This suggests that at high CS concentrations something is inhibiting binding of CS to the nanoemulsion, but not for PLL.

The data in Figure 5 were used to calculate the binding constant $\mathrm{K}_{\mathrm{b}}$ (Figure 6) for the coating using the equation 2.

For PLL the binding constant is quite small compared to CS, and is relatively constant as a function of concentration of material used. At
B

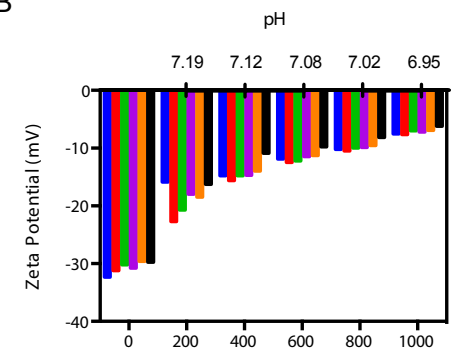

D

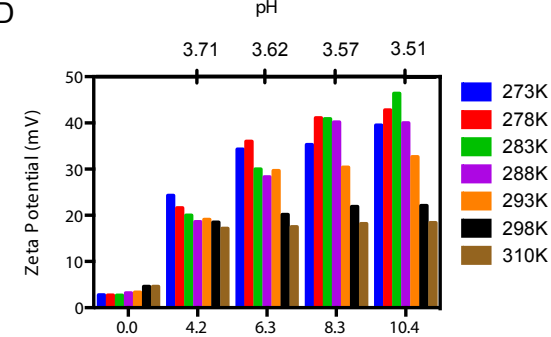

Concentration $(\mu \mathrm{M})$

Figure 3: Diameter and zeta potential of coated LDNE at various $\mathrm{pH}(\mathrm{A}, \mathrm{B}) \mathrm{PLL}$ coated emulsion (C, D) CS coated emulsion. For Figure $\mathrm{B}$, the zeta potential was measured at $\mathrm{pH} 7.10$ For Figure $\mathrm{D}$, the zeta potential was measured at $\mathrm{pH} 3.60$ Based on Figure 2, size/zeta potential would change a lot when $\mathrm{pH}$ in the range of $6.95 \sim 7.19$ and 3.51 3.71, hence, size/zeta potential of un-coated emulsion was measured at pH 7.1 and 3.6 respectively. The PLL coated emulsion at 310K could not be separated by the centrifuge easily, thus it was difficult to determine the unbound PLL concentration. 


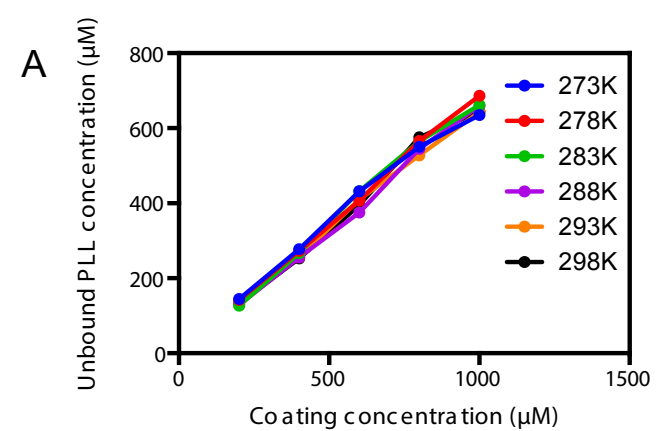

$\mathrm{B}$

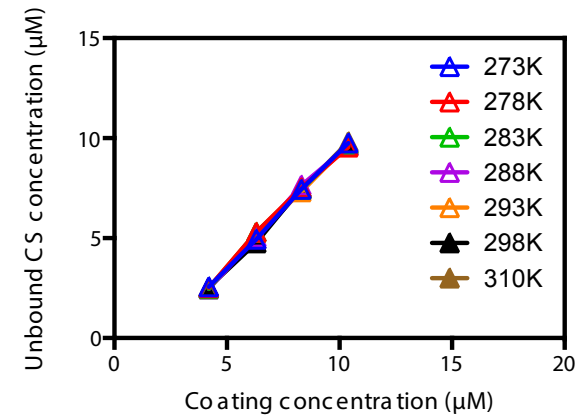

Figure 4: (A) Unbound PLL concentration and (B) unbound CS concentration were measured by colorimetric determination.
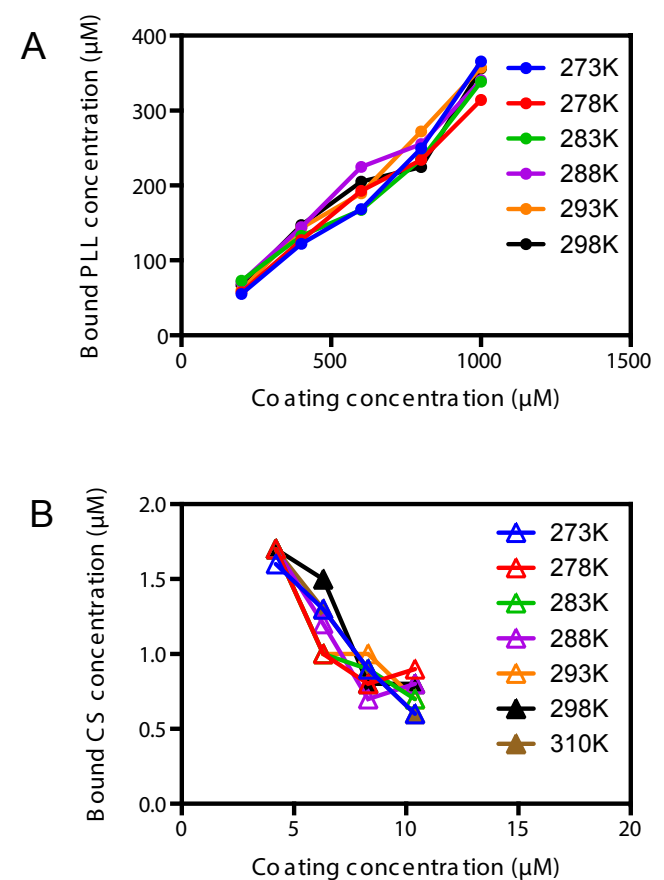

Figure 5: Bound PLL and CS concentration as a function of concentration used at different temperatures.

highest concentration the binding constant seems to increase slightly and this may be due to the fact that by this point the nanoemulsions surfaces are completely coated with PLL and that additional PLL is interacting better with a PLL surface, or possibly that PLL aggregates are beginning to form.
For CS the binding constant is much higher but decreases strongly as the concentration of CS used is increased. The reason why the binding constant is much higher for CS is that the longer chain of CS with about 837.2 units has higher electrostatic attraction than PLL (about 8.6 units). The decrease in binding of CS molecules as more are added indicates that the CS already on the nanoemulsion surface is blocking additional CS from binding.

Based on Eq. 2 (Van't Hoff eq.) the enthalpy $(\Delta \mathrm{H}, \mathrm{kJ} / \mathrm{mol})$ and entropy $(\Delta \mathrm{S}, \mathrm{kJ} / \mathrm{mol} / \mathrm{K})$ can be deduced from the intercept and slope, respectively, and are given in Table 3 and Figure 7.

We find that $\Delta \mathrm{G}$ becomes less negative as CS concentration is increased, but is steady or becomes more negative as more PLL is used. This suggests that additional addition of CS is increasingly unfavored, is more favored for PLL.

To this point we showed that coating of the nanoemulsion is possible with both PLL and CS, but that the affinity of CS for the nanoemulsion is less when more CS is used, and higher when PLL is used. Thermodynamic analysis suggest there is a reversal of both enthalpy and entropy in the case of CS, but a steady trend for PLL. Shown in Figure 8 are $\mathrm{Kb}$ values obtained from this data.

A

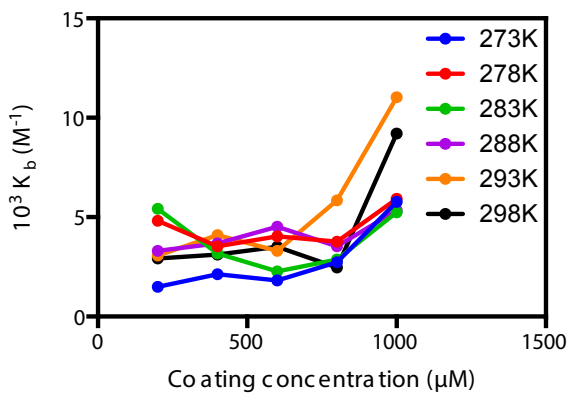

B

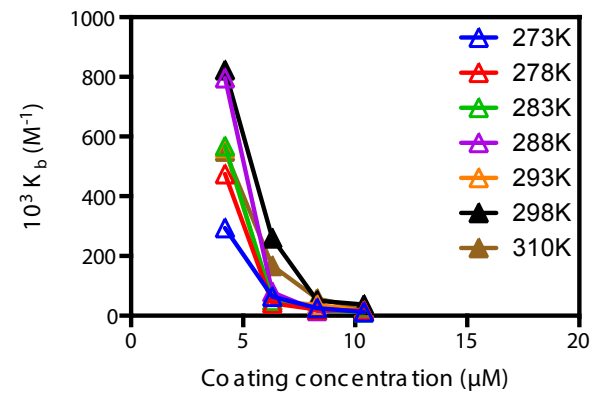

Figure 6: Binding constant, $\mathrm{K}_{\mathrm{b}}$ (a) PLL and (b) $\mathrm{CS}$ at various coating concentration and temperature.

\begin{tabular}{|l|c|c|c|}
\hline \multirow{4}{*}{ PLL } & Conc. $(\boldsymbol{\mu} \mathbf{M})$ & $\boldsymbol{\Delta} \mathbf{H}(\mathbf{k J} / \mathbf{m o l})$ & $\boldsymbol{\Delta S}(\mathbf{J} / \mathbf{m o l} / \mathbf{K})$ \\
\hline \multirow{4}{*}{ CS } & 200 & 8.4 & 95.7 \\
\cline { 2 - 4 } & 400 & 9.0 & 99.2 \\
\cline { 2 - 4 } & 600 & 12.0 & 109.3 \\
\cline { 2 - 4 } & 800 & 1.7 & 74.4 \\
\hline \multirow{3}{*}{} & 1000 & 13.5 & 121.7 \\
\cline { 2 - 4 } & 4.2 & 11.5 & 149.7 \\
\cline { 2 - 4 } & 6.3 & 27.8 & 190.3 \\
\hline & 8.3 & 20.9 & 158.0 \\
\hline
\end{tabular}

Table 3: Thermodynamic parameters of PLL/CS coated emulsions. 
Another way to analyze this data is to estimate the number of molecules attached to the nanoemulsion via the zeta potential measurements and to determine the effective number of particles present. This approach makes the inherent assumption that each charge on the coating molecules neutralizes exactly one charge on the nanoemulsion, and that all samples have the same amount of nanoemulsion.

A

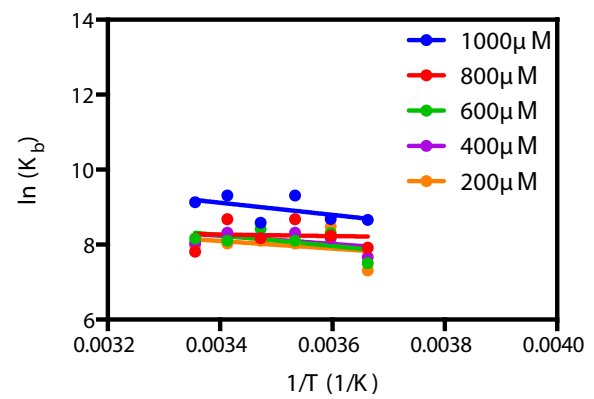

B

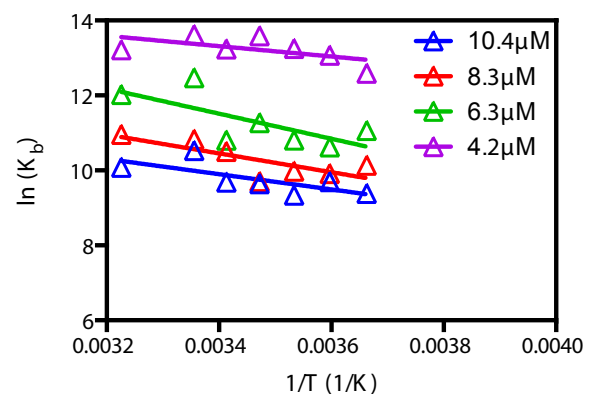

Figure 7: Van't Hoff plot for coating (a) PLL (b) CS.

\section{A}

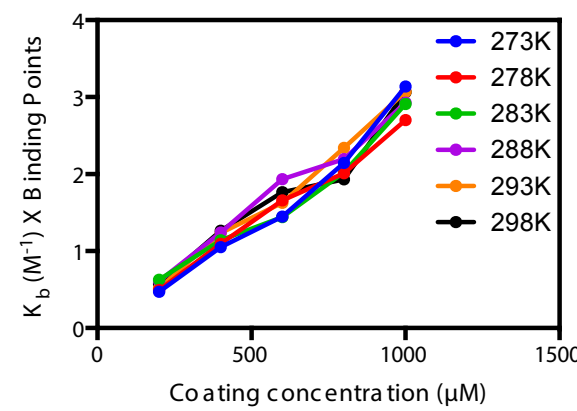

B

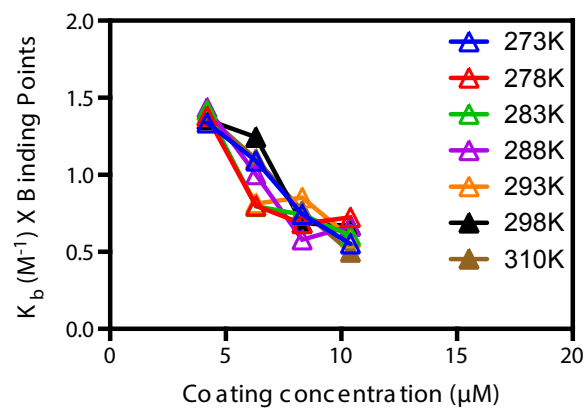

Figure 8: $K_{b}$ for $(A) P L L(B) C S$.
The zeta potential dependence on $\mathrm{pH}$ and temperature is expressed by the following equation [40]:

$$
\frac{d^{2} \psi}{d l^{2}}=-\frac{e}{\varepsilon} \sum_{i} Z_{i} n_{i 0} \exp \left(\frac{Z_{i} e \psi}{k T}\right)
$$

where: $\psi$ is the potential at the distance $l$ from the particle surface

$\varepsilon$ is the dielectric constant of medium

is the bulk concentration of ions with charge $Z_{\mathrm{i}}$

$e$ is one electron charge

$k$ is the Boltzman's constant

$T$ is the absolute temperature

If the colloidal system has low charge density, the quantity $\left(Z_{i} e \psi / k T\right)$ would be less than one and the above equation can be simplified as [40]

$$
\psi(l)=\psi_{\delta} e^{-k l}
$$

where: $\psi_{\delta}$ is the Stern potential

$\kappa$ is the reciprocal of Debye length

As the distance from the particle surface is equal to Debye length, the electric potential would be the zeta potential. In other words, the Stern potential is 2.7 times to the zeta potential according to equation 6 (as $l$ is equal to Debye length). Because the Stern plane is really close to the particle surface, we assume that the value of Stern potential should be close to the surface potential. Moreover, surface charge density can be calculated from the surface potential by applying the following equation,

$$
\sigma=\frac{\varepsilon \varepsilon_{0} \psi_{0}}{\lambda_{d}}
$$

where:

$\sigma$ is the surface charge density

$\varepsilon$ is the dielectric constant of medium $\left(\varepsilon_{\text {water }}=78.5\right)$

$\varepsilon_{0}$ is the vacuum permittivity

$\psi_{0}$ is the surface potential

$\lambda_{d}$ is the Debye length (or $\kappa^{-1}$ )

The surface charge can be obtained by using the area of particle surface so the surface charge change can be calculated after coating. Finally, by using the surface charge change and the charge of each coating material, the amount of coating materials on one emulsion can be derived. Before that, the Debye length is defined as the following equation:

$$
\lambda=\sqrt{\sum \frac{\varepsilon \varepsilon_{0} k T}{Z_{i}^{2} e^{2} n_{i o}}}
$$

The Debye length, Stern potential (viewed as surface potential), surface charge density, surface charge (Q), and surface charge change $(\Delta \mathrm{Q})$ are shown in Table 4.

The calculated number of bound molecules per single emulsion particle is estimated by dividing the change in surface charge by the charge of a single coating molecule in Table 4. PLL with average molecule weight 1250 has 8.6 repeat units per molecule, and CS has 837.2 repeat units per molecule. Hence, assuming each unit has one unit of electrical charge, then one PLL and one CS molecule would have a total charge of $1.37 \times 10^{-18} \mathrm{C}$ and $1.34 \times 10^{-16} \mathrm{C}$ respectively. The number 
Page 7 of 10

\begin{tabular}{|c|c|c|c|c|c|c|}
\hline Temperature (K) & Concentration (UM) & $\lambda_{d}(\mathrm{~nm})$ & $\Psi_{\delta}(\mathrm{mV})$ & $\sigma\left(C / m^{2}\right)$ & $10^{-15} Q(C)$ & ${ }^{10^{-15} \Delta Q(C)}$ \\
\hline \multirow[t]{6}{*}{273} & 0 & 0.746 & -88.0 & -0.818 & -12.1 & 0 \\
\hline & 1000 & 0.746 & -20.6 & -0.019 & -2.8 & 9.3 \\
\hline & 800 & 0.746 & -27.7 & -0.026 & -3.8 & 8.3 \\
\hline & 600 & 0.746 & -32.4 & -0.030 & -4.4 & 7.7 \\
\hline & 400 & 0.746 & -40.2 & -0.037 & -5.5 & 6.6 \\
\hline & 200 & 0.746 & -43.2 & -0.040 & -6.0 & 6.1 \\
\hline \multirow[t]{6}{*}{278} & 0 & 0.753 & -84.8 & -0.078 & -10.3 & 0 \\
\hline & 1000 & 0.753 & -20.8 & -0.019 & -2.5 & 7.8 \\
\hline & 800 & 0.753 & -20.8 & -0.026 & -3.4 & 6.9 \\
\hline & 600 & 0.753 & -34.0 & -0.031 & -4.1 & 6.2 \\
\hline & 400 & 0.753 & -42.4 & -0.039 & -5.2 & 5.1 \\
\hline & 200 & 0.753 & -61.7 & -0.057 & -7.5 & 2.8 \\
\hline \multirow[t]{6}{*}{283} & 0 & 0.760 & -82.2 & -0.075 & -11.4 & 0 \\
\hline & 1000 & 0.760 & -19.1 & -0.017 & -2.7 & 8.7 \\
\hline & 800 & 0.760 & -27.1 & -0.025 & -3.8 & 7.6 \\
\hline & 600 & 0.760 & -33.2 & -0.030 & -4.6 & 6.8 \\
\hline & 400 & 0.760 & -40.2 & -0.037 & -5.6 & 5.8 \\
\hline & 200 & 0.760 & -56.3 & -0.051 & -7.8 & 3.6 \\
\hline \multirow[t]{6}{*}{288} & 0 & 0.767 & -83.7 & -0.076 & -11.4 & 0 \\
\hline & 1000 & 0.767 & -19.6 & -0.018 & -2.7 & 8.7 \\
\hline & 800 & 0.767 & -26.8 & -0.024 & -3.7 & -7.7 \\
\hline & 600 & 0.767 & -31.3 & -0.028 & -4.3 & 7.1 \\
\hline & 400 & 0.767 & -40.0 & -0.036 & -5.4 & 6.0 \\
\hline & 200 & 0.767 & -48.9 & -0.044 & -6.7 & 4.7 \\
\hline \multirow[t]{6}{*}{293} & 0 & 0.774 & -80.5 & -0.072 & -10.6 & 0 \\
\hline & 1000 & 0.774 & -18.8 & -0.017 & -2.4 & 8.2 \\
\hline & 800 & 0.774 & -26.0 & -0.023 & -3.4 & 7.2 \\
\hline & 600 & 0.774 & -30.7 & -0.028 & -4.1 & 6.5 \\
\hline & 400 & 0.774 & -38.1 & -0.034 & -5.0 & 5.6 \\
\hline & 200 & 0.774 & -50.3 & -0.045 & -6.6 & 4.0 \\
\hline \multirow[t]{6}{*}{298} & 0 & 0.780 & -80.8 & -0.072 & -10.5 & 0 \\
\hline & 1000 & 0.780 & -16.9 & -0.015 & -2.2 & 8.3 \\
\hline & 800 & 0.780 & -22.2 & -0.020 & -2.9 & 7.6 \\
\hline & 600 & 0.780 & -26.6 & -0.024 & -3.5 & 7.0 \\
\hline & 400 & 0.780 & -29.6 & -0.026 & -3.9 & 6.6 \\
\hline & 200 & 0.780 & -44.3 & -0.039 & -5.8 & 4.7 \\
\hline \multirow[t]{5}{*}{273} & 0 & 0.746 & 7.6 & 0.007 & 0.9 & 0 \\
\hline & 10.4 & 0.746 & 107.4 & 0.100 & 13.4 & 12.5 \\
\hline & 8.3 & 0.746 & 96.0 & 0.089 & 11.9 & 11.0 \\
\hline & 6.3 & 0.746 & 93.2 & 0.086 & 11.6 & 10.7 \\
\hline & 4.2 & 0.746 & 66.1 & 0.061 & 8.2 & 7.3 \\
\hline \multirow[t]{5}{*}{278} & 0 & 0.753 & 7.4 & 0.007 & 0.8 & 0 \\
\hline & 10.4 & 0.753 & 116.3 & 0.107 & 13.5 & 12.7 \\
\hline & 8.3 & 0.753 & 111.7 & 0.103 & 12.9 & 12.1 \\
\hline & 6.3 & 0.753 & 97.9 & 0.090 & 11.3 & 10.5 \\
\hline & 4.2 & 0.753 & 58.7 & 0.054 & 6.8 & 6.0 \\
\hline \multirow[t]{5}{*}{283} & 0 & 0.760 & 7.3 & 0.007 & 0.9 & 0 \\
\hline & 10.4 & 0.760 & 126.1 & 0.115 & 15.2 & 14.3 \\
\hline & 8.3 & 0.760 & 111.2 & 0.102 & 13.4 & 12.5 \\
\hline & 6.3 & 0.760 & 81.6 & 0.075 & 9.8 & 8.9 \\
\hline & 4.2 & 0.760 & 54.4 & 0.050 & 6.5 & 5.6 \\
\hline \multirow[t]{5}{*}{288} & 0 & 0.767 & 8.63 & 0.008 & 1.0 & 0 \\
\hline & 10.4 & 0.767 & 108.7 & 0.099 & 13.1 & 12.1 \\
\hline & 8.3 & 0.767 & 109.3 & 0.099 & 13.2 & 12.2 \\
\hline & 6.3 & 0.767 & 76.9 & 0.070 & 9.3 & 8.3 \\
\hline & 4.2 & 0.767 & 50.6 & 0.046 & 6.1 & 5.1 \\
\hline \multirow[t]{5}{*}{293} & 0 & 0.773 & 9.2 & 0.008 & 1.2 & 0 \\
\hline & 10.4 & 0.773 & 88.9 & 0.080 & 11.4 & 10.2 \\
\hline & 8.3 & 0.773 & 82.6 & 0.074 & 10.6 & 9.4 \\
\hline & 6.3 & 0.773 & 80.7 & 0.073 & 10.4 & 9.2 \\
\hline & 4.2 & 0.773 & 51.9 & 0.047 & 6.7 & 5.5 \\
\hline
\end{tabular}




\begin{tabular}{|c|c|c|c|c|c|c|}
\hline \multirow[t]{5}{*}{298} & 0 & 0.780 & 12.6 & 0.011 & 1.6 & 0 \\
\hline & 10.4 & 0.780 & 60.1 & 0.054 & 7.5 & 5.9 \\
\hline & 8.3 & 0.780 & 59.5 & 0.053 & 7.4 & 5.8 \\
\hline & 6.3 & 0.780 & 54.9 & 0.049 & 6.8 & 5.2 \\
\hline & 4.2 & 0.780 & 50.3 & 0.045 & 6.3 & 4.7 \\
\hline \multirow[t]{5}{*}{310} & 0 & 0.795 & 12.5 & 0.011 & 2.0 & 0 \\
\hline & 10.4 & 0.795 & 50.0 & 0.044 & 8.1 & 6.1 \\
\hline & 8.3 & 0.795 & 49.5 & 0.043 & 8.0 & 6.0 \\
\hline & 6.3 & 0.795 & 47.6 & 0.042 & 7.7 & 5.7 \\
\hline & 4.2 & 0.795 & 46.8 & 0.041 & 7.6 & 5.6 \\
\hline
\end{tabular}

Table 4: The Debye length, Stern potential, surface charge density, surface charge and surface charge change for PLL/CS coated emulsions.
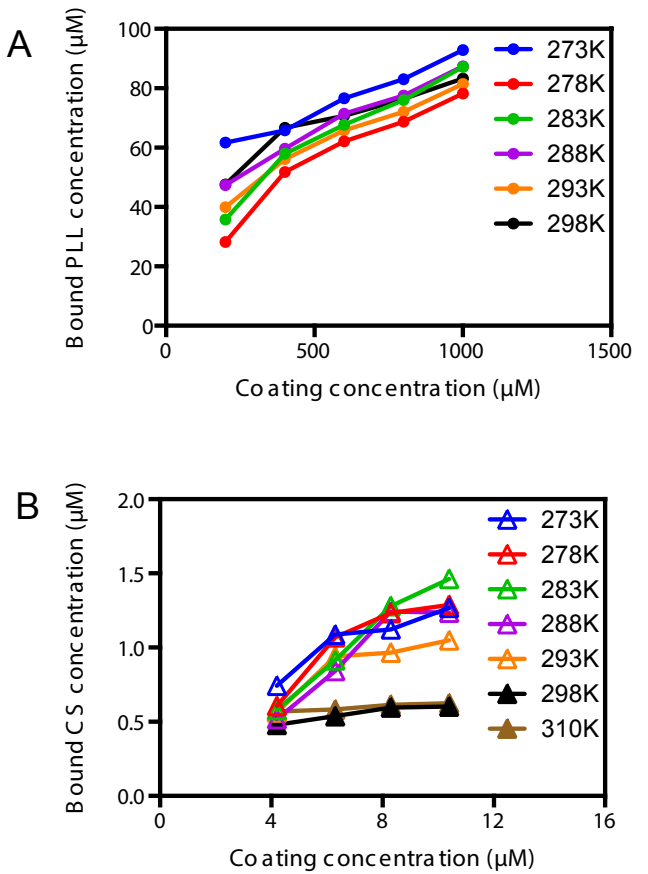

Figure 9: Calculated bound concentrations of coating materials on tota nanoemulsion at different temperatures (A) PLL and (B) CS.

of bound PLL/CS on one nanoemulsion can be calculated. Besides, the theoretical nanoemulsion concentration is estimated $68.4 \mathrm{nmol}$ so the number of bound PLL/CS on total nanoemulsion can be acquired. The results of total bound PLL/CS concentration can be viewed in Figure 8.

Comparing Figure 9 with Figure 6 we find a discrepancy. We can explain these results by an expansion or compression of the specific volume of coating molecules as they accumulate on the emulsion surface. A compression and expansion ratio (CER) can be defined:

$$
C E R=\frac{\text { Bound concentration by colorimetric determination }}{\text { Bound concentration by theoretical calculation }}
$$

The numerator and the denominator are taken from the data in Figures 6 and 8 respectively. If CER is larger than one, it means the coating materials are compressed. If CER is smaller than one, it means the coating materials on emulsion surface expand. In Figure 9 the CER values for PLL and CS coated emulsions are plotted against the amount of PLL and CS used in the coating procedure.

Figure 10 suggests PLL molecules become more compressed and their packing density increases as more PLL is used. In contrast CS expands when more CS is used. In general temperature does not appear to affect the CER of either material greatly. These observations
A

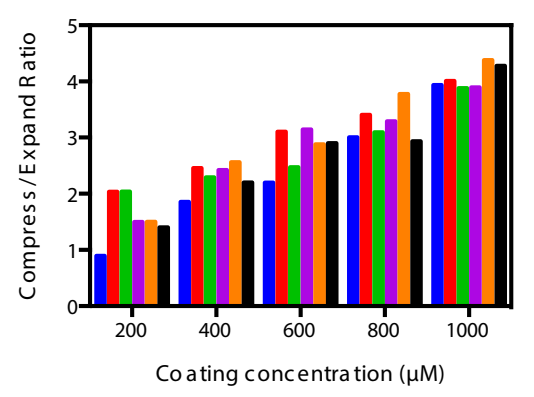

B

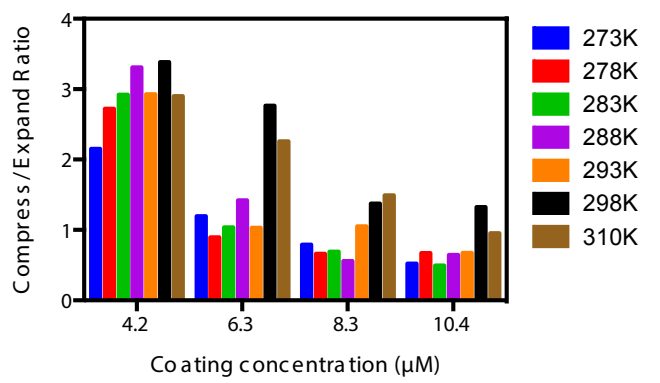

Figure 10: Temperature and coating concentration-dependent $C E R$ values (defined by equation 9) (A) PLL, (B) CS. As PLL concentration increases, PLL molecules on the emulsion surface become more compressed. In contrast, $\mathrm{CS}$ molecules on the emulsion surface become more expanded as CS concentration increases.

are consistent with the bulk observation that protein specific volume decreases with higher protein concentration and the idea of macromolecular (like polysaccharide) crowding is also similar, which high concentration of protein alters its own conformation $[41,42]$

To confirm this hypothesis we performed fluorescence quenching tests to obtain independent confirmation of the density of the coating layer. Pyrene-doped PFOB emulsions coated with PLL and CS at different levels were exposed to 5, 10, 20 and $50 \mathrm{mM}$ solutions of the fluorescence quencher KI to form mixtures with final concentrations of 3.75, 7.5, 15 and $37.5 \mathrm{mM}$. For coated emulsions with CER values below 1, iodide ions should be able to pass through the coating and interact with the pyrene at the emulsion/coating interface leading to measureable fluorescence quenching. For those with CER values much higher than 1, iodide-induced quenching should be impeded.

Figure 11 shows that the ratio of $\mathrm{I}_{0} / \mathrm{I}$ for KI quenching of pyrene in the PLL emulsions increases linearly with iodide quencher concentration. For PLL the quenching as a function of coating amount is what is expected if the coating becomes denser as more material is deposited. For CS-coated emulsions there is more quenching when a larger amount of CS is attached to the particles. This represents an unusual behavior, but does make sense in the context of the results 
of thermodynamics trend deduced from the data. However, for the highest KI concentration, the overall viscosity of the suspension increased so that the diffusion of iodide ions was compromised slowed so there is less quenching possible.

A Stern-Volmer constant $\left(\mathrm{K}_{\mathrm{s}-\mathrm{V}}\right)$ can be acquired from the slopes of the trend lines (linear regression) in Figure 11 (Table 5). The trend lines of the ratio of $\mathrm{I}_{0} / \mathrm{I}$ versus [Q] for CS coated emulsion only are determined by the KI concentrations: 3.75, 7.5 and $15 \mathrm{mM}$. The bimolecular quenching rate constant $\left(\mathrm{k}_{\mathrm{q}}\right)$ can also be deduced using the pyrene fluorescent excited state lifetime $\left(\tau_{0}\right)$ in air-equilibrated water, $126 \mathrm{~ns}$. The reason may be that CS coated emulsion surface has positive charges to attract the negatively charged iodide ions but PLL coated emulsion surface has negative charges, shown in Figure 2.

Based on the result of CER ratio calculation shown in Figure 9 and fluorescent quenching results, we can propose a concept for PLL and CS molecule conformation change during emulsion coating (Figure 12).

\section{Conclusions}

This work shows an analysis of an emulsion coating process that in addition to providing information about the thermodynamics of

A

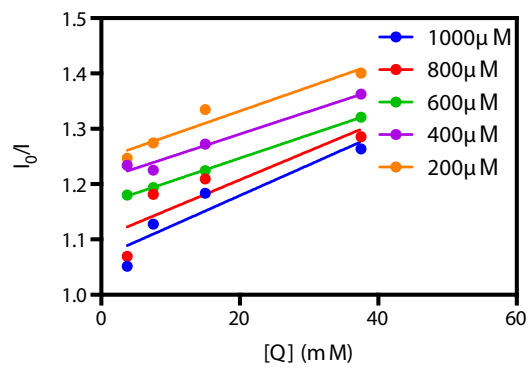

B

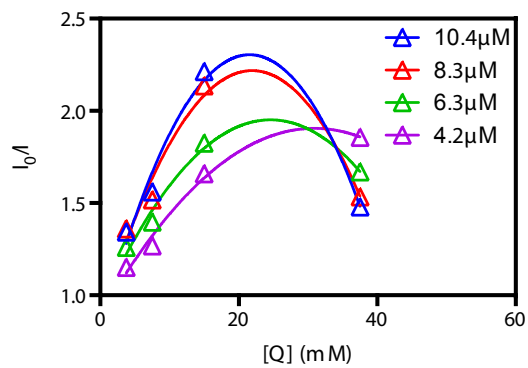

Figure 11: The ratio of the initial fluorescent intensity to the final fluorescent intensity versus the quencher $(\mathrm{Q})$ concentration (i.e. $\mathrm{KI}$ ): a) PLL-coated emulsions, b) CS-coated emulsions.

\begin{tabular}{|c|c|c|c|c|}
\hline Conc. $(\boldsymbol{\mu M})$ & Trend line slope & $\mathbf{R}^{\mathbf{2}}$ & $\mathbf{K}_{\mathbf{s - v}}\left(\mathbf{M}^{-\mathbf{1}}\right)$ & $\mathbf{1 0}^{-\mathbf{9}} \mathbf{k}_{\mathbf{q}}\left(\mathbf{M}^{-\mathbf{1}} \mathbf{S}^{-\mathbf{1}}\right)$ \\
\hline 1000 & 0.00556 & 0.8788 & 5.6 & 0.044 \\
\hline 800 & 0.00523 & 0.7780 & 5.2 & 0.042 \\
\hline 600 & 0.00421 & 0.9995 & 4.2 & 0.033 \\
\hline 400 & 0.00411 & 0.9745 & 4.1 & 0.033 \\
\hline 200 & 0.00437 & 0.9369 & 4.4 & 0.035 \\
\hline 10.4 & 0.08018 & 0.9918 & 80.2 & 0.636 \\
\hline 8.3 & 0.07078 & 0.9806 & 70.8 & 0.562 \\
\hline 6.3 & 0.05123 & 0.9899 & 51.2 & 0.407 \\
\hline 4.2 & 0.04614 & 0.9870 & 46.1 & 0.366 \\
\hline
\end{tabular}

Table 5: Stern-Volmer constants $\left(\mathrm{K}_{\mathrm{s}-\mathrm{v}}\right)$ and bimolecular quenching rate constant $\left(k_{q}\right)$ of coated emulsion in various concentrations of $\mathrm{KI}$ solution.

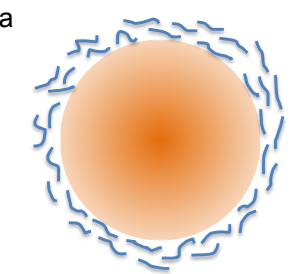

Low PLL coating concentration

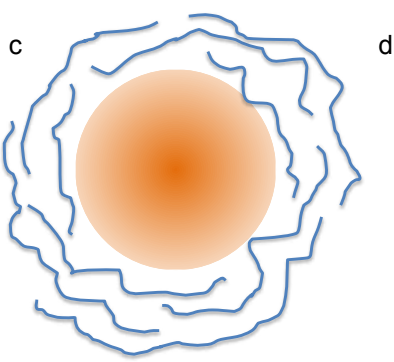

Low CS coating concentration

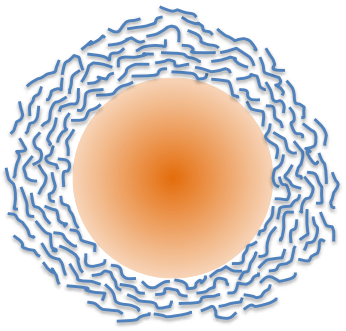

High PLL coating concentration

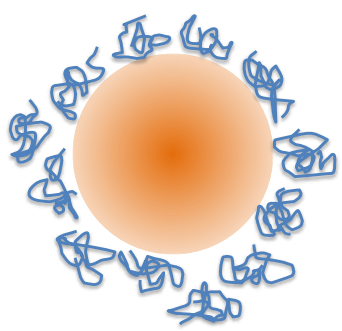

High CS coating concentration
Figure 12: (a) PLL coated emulsion has more expanded surface during low coating concentration. (b) PLL coated emulsion has more compressed surface during high coating concentration. (c) CS coated emulsion has more compressed surface during low coating concentration. (d) CS coated emulsion has more expanded surface during high coating concentration.

coating reveal something fundamental about the characteristics of the coating molecules as the particles are formed. The ionic surfactant DOPA was used to create a negative surface charge on an emulsion make from $98 \%$ non-ionic surfactant lecithin. One formulation with maximum surface charge and minimum modification (2\% DOPA and 98\% lecithin $\mathrm{PFOB}$ emulsion prepared at $\mathrm{pH} 7$ ) are for measurement of binding equilibria as a function of temperature and coating formation, and for zeta potential measurements as a function of coating formation. PLL and CS exhibited different coating characteristics explained by concentration and $\mathrm{pH}$ dependent conformational differences. By using the measurements to back calculate the effective emulsion particle concentration, it is possible to discern a change in the specific volume of the coating molecules that follows the expected trend for protein and sugar molecules in concentrated solution. The trend is rationalized due the high local concentration of these molecules as they accumulate and attach to the emulsion surface. PLL molecules tend to compress on the coated emulsions as the coating concentration increases. Conversely, CS molecules tend to expand as the concentration increases. The effect of compression versus expansion was further validated by means of fluorescent quenching. The ratio of initial fluorescent intensity to final intensity is higher for lower PLL coating concentration and higher CS coating concentration supporting the hypothesis that at these two extreme concentration ranges both conformations of the coating materials are expanded and create a more porous coating that can easily be permeated by small charged ions. Thermodynamic calculations also support this picture.

\section{References}

1. Takeuchi $\mathrm{H}$, Yamamoto $\mathrm{H}$, Kawashima $\mathrm{Y}$ (2001) Mucoadhesive nanoparticulate systems for peptide drug delivery. Advanced Drug Delivery Reviews 47: 39-54.

2. Takeuchi H, Yamamoto H, Niwa, T, Kawashima $Y$ (2001) Enteral absorption of insulin in rats from mucoadhesive chitosan-coated liposomes. Pharmaceutical Research 13: 896-901. 
3. Lee H, Eckmann D, Lee, D, Hicock N, Composto R (2011) Symmetric pHdependent swelling and antibacterial properties of chitosan brushes. Langmuir 27: $12458-12465$.

4. Kaneda M, Caruthers S, Lanza G, Wickline S (2009) Perfluorocarbon nanoemulsions for quantitative molecular imaging and targeted therapeutics. Annals of Biomedical Engineering 37: 1922-1933.

5. McClements D (2010) Design of Nano-Laminated Coatings to Control Bioavailability of Lipophilic Food Components. Journal of Food Science 75: R30-R42.

6. Barea M, Jenkins M, Gaber M, Bridson R (2010) Evaluation of liposomes coated with a $\mathrm{pH}$ responsive polymer. International Journal of Pharmaceutics 402: 89-94.

7. Jerobin J, Sureshkumar R, Anjali C, Mukherjee A, Chandrasekaran N (2012) Biodegradable polymer based encapsulation of neem oil nanoemulsion for controlled release of Aza-A. Carbohydrate Polymers 90: 1750-1756

8. Ohguchi Y, Kawano K, Hattori Y, Maitani Y (2008) Selective delivery of folatePEG-linked, nanoemulsion-loaded aclacinomycin A to KB nasopharyngea cells and xenograft: effect of chain length and amount of folate-PEG linker. Journal of Drug Targeting 16: 660-667.

9. Soppimath K, Aminabhavi T, Kulkarni A, Rudzinski W (2001) Biodegradable polymeric nanoparticles as drug delivery devices. Journal of Controlled Release 70: 1-20.

10. Constantinides $P$, Chaubal M, Shorr R (2008) Advances in lipid nanodispersions for parenteral drug delivery and targeting. Advanced Drug Delivery Reviews 60: 757-767.

11. Koo O, Rubinstein I, Onyuksel H (2005) Role of nanotechnology in targeted drug delivery and imaging: a concise review. Nanomedicine: Nanotechnology, Biology and Medicine 1: 193-212.

12. Billstein P, Carlsson U, Jonsson B, Olofson G, Hook F, et al. (1999) Conformation of human carbonic anhydrase II variants adsorbed to silica nanoparticles. Langmuir 15: 6395-6399.

13. Lundqvist M, Sethson I, Johnson B (2004) Protein adsorption onto silica nanoparticles: conformational changes depend on the particles' curvature and the protein stability. Langmuir 20: 10639-10647.

14. Shang L, Wang $Y$, Jiang J, Dong S (2007) pH-dependent protein conformational changes in albumin: gold nanoparticle bioconjugates: a spectroscopic study. Langmuir 23: 2714-2721.

15. Nguyen T, Doan V, Schwartz B (1999) Conjugated polymer aggregates in solution: Control of interchain interactions. The Journal of Chemical Physics 110: $4068-4078$

16. Tuomi T, Rowley M, Knowles W, Chen Q, McAnally T, et al. () Autoantigenic properties of native and denatured glutamic acid decarboxylase: evidence for a conformational epitope. Clinical Immunology and Immunopathology 71: 53-59.

17. Itoh Y, Itoh M, Frank M, Reichlin M (1992) Autoantibodies to the Ro/SSA autoantigen are conformation dependent II: antibodies to the denatured form of $52 \mathrm{kD}$ Ro/SSA are a cross reacting subset of antibodies to the native $60 \mathrm{kD} \mathrm{Ro}$ SSA molecule. Autoimmunity 14: 89-95

18. Reich N, Sarnow P, Duprey E, Levine A (1983) Monoclonal antibodies which recognize native and denatured forms of the adenovirus DNA-binding protein. Virology 128: 480-484.

19. Elwing H, Nilsson K, Svensson A, Askendahl A, Nilsson U, et al. (1988) Conformational changes of a model protein (complement factor 3 ) adsorbed on hydrophilic and hydrophobic solid surfaces. Journal of Colloid and Interface Science 125: 139-145.

20. Glabe C (2004) Conformation-dependent antibodies target diseases of protein misfolding. Trends in Biochemical Sciences 29: 542-547.

21. Schurr J (1977) Dynamic light scattering of biopolymers and biocolloids. CRC critical reviews in Biochemistry 4: 371-431.

22. Tanner R, Herpigny B, Chen S, Rha C (1982) Conformational change of protein sodium dodecylsulfate complexes in solution: A study of dynamic light scattering. The Journal of Chemical Physics 76: 3866-3872.

23. Dathe M, Fabian H, Gast K, Zirwer D, Winter R, et al. (1996) Conformationa differences of bovine and human corticotrophin releasing hormone A CD, IR,
NMR and dynamic light scattering study. International Journal of Peptide and Protein research 47: 383-393.

24. Haris P, Chapman D (1995) The conformational analysis of peptides using Fourier transform IR spectroscopy. Biopolymers 37: 251-263.

25. Payne K, Veis A (1988) Fourier transform IR spectroscopy of collagen and gelatin solutions: deconvolution of the amide I band for conformational studies. Biopolymers 27: 1749-1760.

26. Kister G, Cassana G, Vert M (1998) Effects of morphology, conformation and configuration on the IR and Raman spectra of various poly (lactic acid). Polymer 39: 267-273.

27. Smith G, Yoon D, Jaffe R, Colby R, Krishnamoorti R, et al. (1996) Conformations and structures of poly (oxyethylene) melts from molecular dynamics simulations and small-angle neutron scattering experiments. Macromolecules 29: 3462 3469 .

28. Kirste R, Ohm H (1985) The conformation of liquid-crystalline polymers as revealed by neutron scattering. Die Makromolekulare Chemie, Rapid Communications 6: 179-185.

29. Lieser G, Fischer E, Ibel K (1975) Conformation of polyethylene molecules in the melt as revealed by small-angle neutron scattering. Journal of Polymer Science: Polymer Letters Edition 13: 39-43.

30. Shen W, Yang D, Ryser H (1984) Colorimetric determination of microgram quantities of polylysine by trypan blue precipitation. Analytical Biochemistry 142: $521-524$

31. Grotzky A, Manaka Y, Fornera S, Willeke M, Walde P (2010) Quantification of a-polylysine: a comparison of four UV/Vis spectrophotometric methods. Analytical Methods 2: 1448-1455.

32. Wischke A, Borchert $H$ (2006) Increased sensitivity of chitosan determination by a dye binding method. Carbohydrate Research 341: 2978-2979.

33. Abou-Shoer M (2010) A Simple Colorimetric Method for the Evaluation of Chitosan. American Journal of Analytical Chemistry 1: 91-94.

34. Muzzarelli R (1998) Colorimetric determination of chitosan. Analytical Biochemistry 260: 255- 257.

35. Schubert K, Kaler E (1994) Microemulsifying fluorinated oils with mixtures of fluorinated and hydrogenated surfactants. Colloids and Surfaces $A$ Physicochemical and Engineering Aspects, 84: 97-106.

36. Kalyanasundaram K (1988) Pyrene fluorescence as a probe of fluorocarbon micelles and their mixed micelles with hydrocarbon surfactants. Langmuir 4 942-945.

37. Acree W, Abraham M (2002) Solubility predictions for crystalline polycyclic aromatic hydrocarbons (PAHs) dissolved in organic solvents based upon the Abraham general solvation mode. Fluid Phase Equilibria 201: 245-258.

38. Lakowicz R (1999) Principles of Fluorescence Spectroscopy. Springer, New York, USA.

39. Birdi K (2008) Handbook of Surface and Colloid Chemistry. (3 ${ }^{\text {rd }}$ edtn) CRC Press Boca Raton Florida.

40. Van den Berg B, Ellis R, Dobson C (1999) Effects of macromolecular crowding on protein folding and aggregation. The EMBO Journal 18: 6927-6933.

41. Homouz D, Perham M, Samiotakis A, Cheung M, Wittung-Stafshede P (2008) Crowded, cell-like environment induces shape changes in aspherical protein Proceedings of the National Academy of Sciences 105: 11754-11759.

42. Geiger M, Turro N (1975) Pyrene fluorescence lifetime as a probe for oxygen penetration of micelles. Photochemistry and photobiology 22: 273-276. 